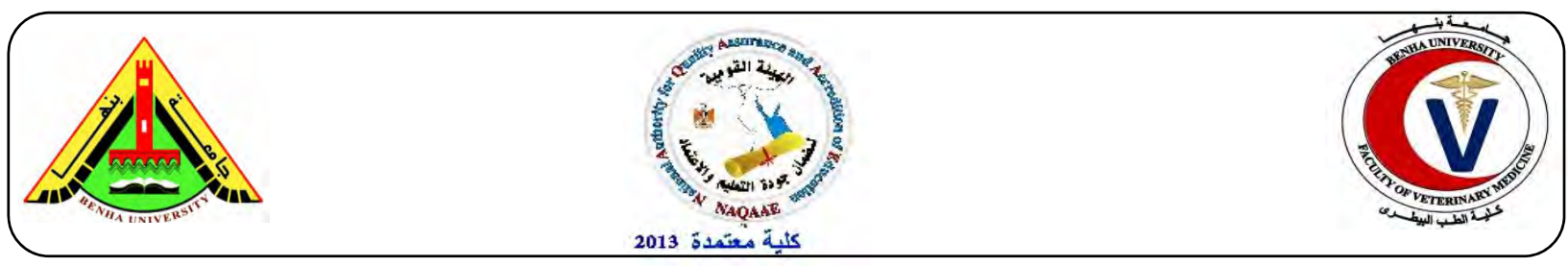

\title{
Bacteriological and Molecular Identification of Campylobacter Species in Chickens and Humans, at Zagazig City, Egypt
}

\author{
Ashraf A. Abd El-Tawab ${ }^{1}$, Ahmed M. Ammar ${ }^{2}$, Heba A. Ahmed ${ }^{3}$, Fatma I. El Hofy ${ }^{1}$, Ahmed A. \\ Hefny $^{4^{*}}$ \\ ${ }^{1}$ Bacteriology, Immunology and Mycology Dep., Fac. Vet. Med. Banha University, ${ }^{2}$ Bacteriology, \\ Immunology and Mycology Dep., Fac. Vet. Med. Zagazig University, ${ }^{3}$ Zoonoses Dep., Fac. Vet. Med. \\ Zagazig University, ${ }^{4}$ Veterinary Hospital, Fac. Vet. Med. Zagazig University
}

\begin{abstract}
A B S T R A C T
The genus Campylobacter is of great importance to public health because it includes several species that may cause diarrhea. Poultry and poultry products are known as important sources of human campylobacteriosis. A total of 533 samples from broiler chickens ;131 cloacal swabs, 39 chicken skin, 39 chicken cecal parts and 78 chicken meat thigh and breast meat (39 of each) were obtained from retail outlets, as well as, 246 stool swabs from persons attending the outpatient clinic of Al-Ahrar public hospital were examined. The isolation rate of Campylobacter species from chicken skin, thigh meat, breast meat, cecal parts, cloacal swabs and human stool samples were $30.8 \%, 38.5 \%, 30.8 \%, 41 \%$, $35.1 \%$ and $5.3 \%$, respectively. The conventional biochemical tests were used for discrimination between C. jejuni and other Campylobacter species based on standardized hippurate hydrolysis test. C. jejuni was isolated from cloacal swabs, skin, thigh meat, breast meat, cecal parts and human stool samples with the isolation rate of $45.7 \%, 50 \%, 46.7 \%, 41.7 \%, 81.3 \%$ and $76.9 \%$, respectively. Real-Time PCR targeting hipO gene specific for C. jejuni was used for the confirmation of phenotypically identified 31 C. jejuni isolates. The results showed that the conventional culture methods and biochemical reactions were $100 \%$ in accordance with the results of PCR for identification and differentiation of $C$. jejuni.
\end{abstract}

Keywords: C. jejuni, C. coli, chickens, humans, RT-PCR, Egypt

(http://www.bvmj.bu.edu.eg)

(BVMJ-28(1): 17-26, 2015)

\section{INTRODUCTION}

C ampylobacter species are Gramnegative bacteria within the family Campylobacteraceae that require microaerobic growth conditions. Thermophilic Campylobacters, including C. jejuni and C. coli that are the most frequent Campylobacter species isolated from patients with diarrhea in both industrial and developing countries (Rahimi and Ameri, 2011). Campylobacter species are widespread in nature prevailing mainly in the alimentary tract of wild and domesticated birds and mammals. Transmission to humans is most commonly through consumption and handling of chicken meat products contaminated with this zoonotic pathogen during slaughtering and carcass processing (EFSA, 2010).Conventional biochemical tests for discrimination between C. jejuni and C. coli depend mainly on hippurate hydrolysis which is the only phenotypic test for differentiation between the two species. However, both false positive and false negative results have been reported (Waino et al., 2003). Therefore, molecular identification methods have been described as an alternative to the inaccurate, time consuming, biochemical phenotypic methods (LaGier et al., 2004). The aim of the current work was to investigate the contribution of chicken as a potential source of Campylobacter species particularly $C$. 
jejuni infections in humans by using conventional and molecular tools.

\section{MATERIAL AND METHODS}

\subsection{Samples}

A total of 533 samples from broiler chickens at 6 weeks age; 131 cloacal swabs, 39 chicken skin, 39 cecal parts and 78 chicken meat (thigh and breast meat, 39 of each) were obtained from retail outlets at Zagazig, Egypt. Moreover, 246 stool swabs collected from persons attending the outpatient clinic of Al-Ahrar public hospital, Zagazig city, Sharkia Province, Egypt, were examined.

\subsection{Samples preparation}

\subsubsection{Stool and cloacal swabs}

Sterile swabs were inserted into the cloaca and voided human stool samples and then directly immersed into sterile Preston enrichment broth base containing Campylobacter growth supplement (Ellerbroek et al., 2010).

\subsubsection{Skin, cecal and meat samples}

Twenty five grams from each incised skin, cecal parts and chicken meat (thigh and breast) were aseptically transferred to a sterile blender containing $225 \mathrm{ml}$ of Preston enrichment broth for homogenization of the sample (Sallam, 2001).

\subsection{Bacteriological examination}

\subsubsection{Isolation of Campylobacter species}

The collected samples in Preston enrichment broth were incubated at $42^{\circ} \mathrm{C}$ for 24-48 hours with less than $1 \mathrm{~cm}$ of headspace left in the culture vessel with tightly capped lids (Oxoid, 2006). After enrichment, $0.1 \mathrm{ml}$ of the broth was streaked onto modified Campylobacter selective agar base Cefoperazone Charcoal Desoxycolate Agar (mCCDA) containing CCDA Selective Supplement. The plates were then incubated at $42^{\circ} \mathrm{C}$ in darkness for 48 hours under microaerophilic conditions

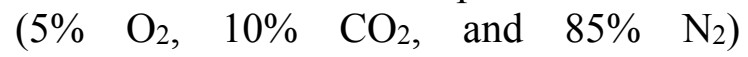

(Vandepitte and Verhaegen, 2003).

2.3.2. Preliminary confirmation of
thermophilic Campylobacter
species

Thermophilic Campylobacter species were preliminary identified by their colonial morphology on mCCDA media. Suspected colonies were purified on blood agar plates and subjected to Gram staining, motility test, growth at $25^{\circ} \mathrm{C}$ and $41.5^{\circ} \mathrm{C}$ and oxidase test (ISO, 2006).

\subsubsection{Biochemical identification of Campylobacters}

The preliminary identified Campylobacter species were further subjected to catalase test, susceptibility to nalidixic acid and cephalothin and rapid hippurate hydrolysis test (Nachamkin, 1999).

\subsection{Molecular identification of C. jejuni}

\subsubsection{DNA extraction}

DNA extraction from the biochemically identified isolates was performed according to the manufacturer guidelines using Bacterial DNA Extraction Kit (Spin-column) (BioTeke Corporation, China).

\subsubsection{Real-Time probe based PCR}

A Real-Time probe based quantitative PCR (qPCR) reaction was used for the confirmation of 31 C. jejuni isolates. Species-specific primer and TaqMan probe sets targeting hipO gene specific for $C$. jejuni (LaGier et al., 2004) were synthesized (AlphaDNA, Canada). The sequences of primers and probe were $\mathrm{Cj}-\mathrm{F}$ 5'- TGCTAGTGAGGTTGCAAAAGAA TT-3', Cj-R 5'-TCATTTCGCAAA AAAATCCAAA-3' and Cj-FAM 5'ACGATGATTAAATTCACAATTTT TTTCGCC AAA-3'. Non-template DNA and positive controls of C. jejuni, C. coli, E. coli, S. Typhimurium, Staph. aureus and two biochemically identified Campylobacter isolates other than C. jejuni and $C$. coli were also run to determine the specificity of the reaction. 


\section{RESULTS}

\subsection{Preliminary confirmation of thermophilic Campylobacter species}

Campylobacter species were preliminary identified by their colonial morphology on mCCDA and sheep blood agar. C. jejuni on mCCDA appeared as greyish, flat, moistened, with a tendency to spread colonies that may have a metal sheen. However, on $5-7 \%$ sheep blood agar $C$. jejuni had characteristic colonies of oil drop like appearance (translucent droplet-like colonies), slightly pink, round, convex, smooth and shiny, with a regular edge. Occasionally, C. jejuni showed greyish, flat, moistened, with a tendency to spread colonies on sheep blood agar. Campylobacter species were also confirmed by production of oxidase. The suspected Campylobacter organisms in freshly prepared cultures appeared as Gram negative (faint in color) curved, twisted bacilli. In old cultures, or when exposed to air for prolonged time, colonies transformed from spiral form to coccoid morphology. Examination of motility showed that Campylobacters are highly motile with characteristic corkscrew like motility, while in old cultures they were less motile. Moreover, thermophilic Campylobacters did not grow at $25^{\circ} \mathrm{C}$ in a microaerobic atmosphere or at $41.5^{\circ} \mathrm{C}$ aerobically for 48 hours.

\subsection{Identification of Campylobacter species}

For the identification of thermophilic Campylobacters to the species level, catalase test, susceptibility to nalidixic acid and cephalothin and rapid hippurate hydrolysis test were performed on 114 biochemically suspected isolates. The results showed that all Campylobacters were catalase positive, while, most of Campylobacter isolates were resistant to nalidixic acid; therefore, it was difficult to differentiate C. lari and C. coli. C. jejuni was differentiated by rapid $\mathrm{Na}$ hippurate hydrolysis test, formation of dark blue or purple color indicated a positive hippurate hydrolysis (Table 1).

\subsection{Confirmation of C. jejuni by Real-Time PCR}

qPCR targeting hipO gene specific for $C$. jejuni showed that $31 \mathrm{C}$. jejuni isolates were confirmed (Figure 1). The specificity of the reaction was characterized because primer and probe sets specific for $C$. jejuni did not amplify DNA from $C$. coli and other positive controls.

\subsection{Prevalence of Campylobacter species in different collected samples}

According to the phenotypic and biochemical identification, Campylobacter species were isolated from $21.4 \%$ of the examined samples. . The results indicated a high isolation rate of Campylobacter species from chicken ( intestine (41\%), thigh meat (38.5\%), cloacal swabs $(35.1 \%)$ and breast meat $(30.8 \%)$ and neck skin $(30.8 \%)$.

In humans, only $5.3 \%$ of the stool samples were positive for Campylobacter species (Table 2). Identification of the isolated Campylobacter species showed that $C$. jejuni, C. coli / C. lari and C. hyointestinal were identified in $54.4 \%, 42.1 \%$ and $3.5 \%$, respectively. In chicken samples, $C$. jejuni were isolated from intestine, neck skin, thigh meat, cloacal swabs and breast meat with the isolation rates of $81.3 \%, 50 \%$, $46.7 \%, 45.7 \%$ and $41.7 \%$ respectively.

However, the isolation rate of C. coli/C. lari from breast meat, thigh meat, neck skin, cloacal swabs and caecal parts was $58.3 \%$, $53.3 \%, 50 \%, 47.8$ and $12.5 \%$. In humans, $C$. jejuni and C. coli/C. lari were identified from $76.9 \%$ and $23.1 \%$ out of the isolates respectively. C. hyointestinal was only identified with an incidence of $6.5 \%$ and $6.3 \%$ from cloacal swabs and intestine respectively.

\section{DISCUSSION}

Campylobacters are one of the most important food bacteria causing 
Abd El-Tawab et al. (2015)

Table (1): Results of thermophilic Campylobacter identification

\begin{tabular}{lcccccccc}
\hline \multirow{2}{*}{ Test / species } & \multicolumn{2}{c}{ Catalase } & \multicolumn{2}{c}{ Nalidixic acid } & \multicolumn{2}{c}{ Cephalothin } & \multicolumn{2}{c}{ Na hippurate } \\
\cline { 2 - 9 } & + & - & $\mathrm{S}$ & $\mathrm{R}$ & $\mathrm{S}$ & $\mathrm{R}$ & + & - \\
C. jejuni (62) & 62 & 0 & 0 & 62 & 0 & 62 & 62 & 0 \\
C. col i/ C. lari & 48 & 0 & 2 & 46 & 0 & 48 & 0 & 48 \\
(48) & & 0 & 0 & 4 & 4 & 0 & 0 & 4 \\
C. hyointestinal (4) & 4 & 0 &
\end{tabular}

S: sensitive R: resistant

Figure (1): Amplification curve of biochemically suspected $C$. jejuni using probe bared qPCR (1:C. jejuni positive control, 2-12: biochemically suspected $C$. jejuni isolates, 13: Negative controls)

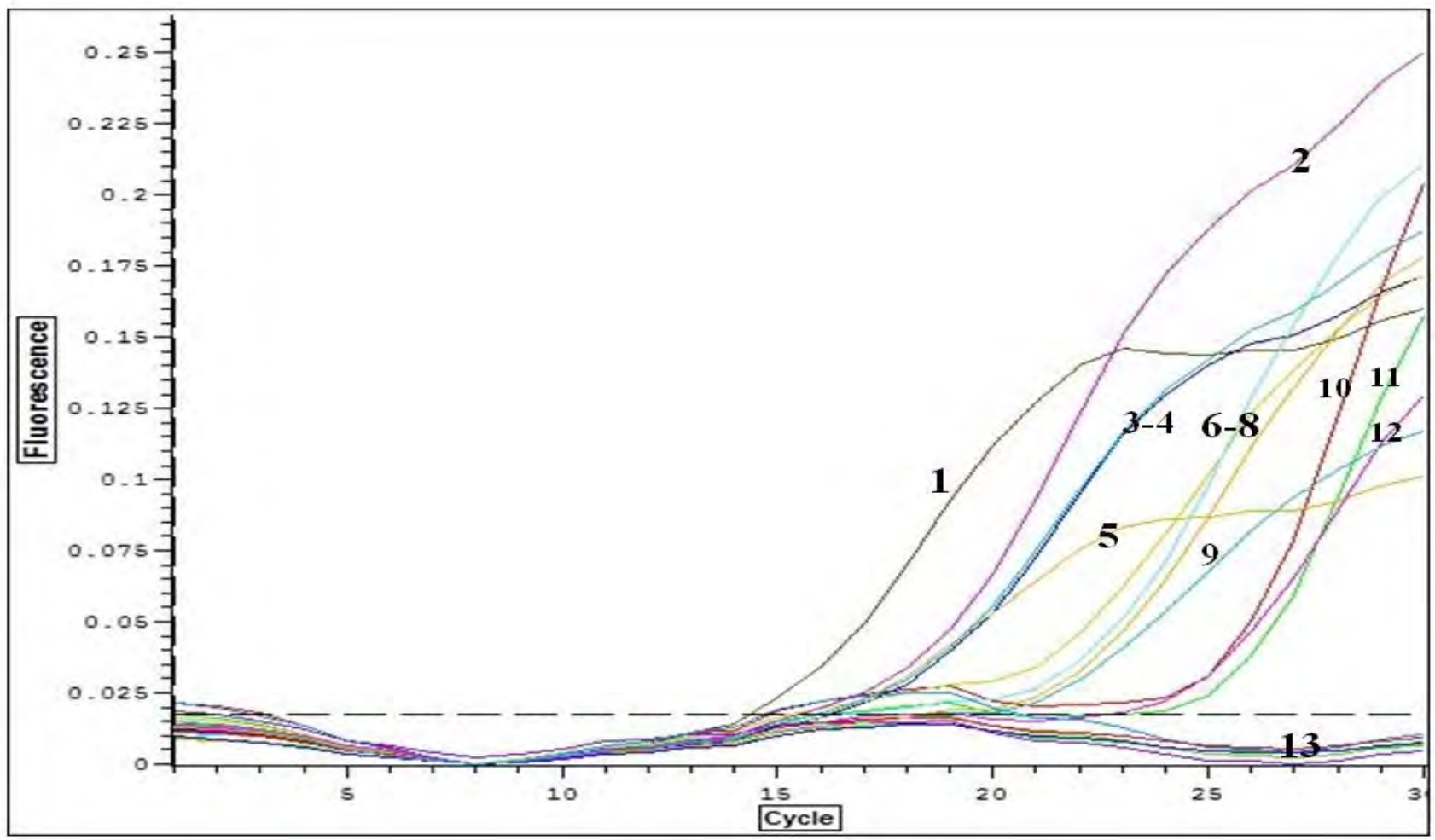


Table (2): Occurrence of different Campylobacter species in the examined samples [Number (proportion, 95\% CI)]

\begin{tabular}{|c|c|c|c|c|c|c|}
\hline \multicolumn{2}{|c|}{ Type of samples } & \multirow{2}{*}{$\begin{array}{c}\begin{array}{c}\text { Number } \\
\text { examined }\end{array} \\
131\end{array}$} & \multirow{2}{*}{$\begin{array}{c}\begin{array}{c}\text { Campylobacter } \\
\text { species* }\end{array} \\
46 \\
(35.1,27-43.9)\end{array}$} & \multirow{2}{*}{$\begin{array}{c}\text { C. jejuni** } \\
21 \\
(45.7,30.9-61)\end{array}$} & \multirow{2}{*}{$\begin{array}{c}\text { C. coli / C. lari** } \\
\begin{array}{c}22 \\
(47.8,32.9-63.1)\end{array}\end{array}$} & \multirow{2}{*}{$\begin{array}{c}\text { C. hyointestinal** } \\
\begin{array}{c}3 \\
(6.5,1.4-17.9)\end{array}\end{array}$} \\
\hline & Cloacal swabs & & & & & \\
\hline & Neck skin & 39 & $\begin{array}{c}12 \\
(30.8,17-47.6)\end{array}$ & $\begin{array}{c}6 \\
(50,21.1-78.9)\end{array}$ & $\begin{array}{c}6 \\
(50,21.1-78.9)\end{array}$ & $\begin{array}{c}0 \\
(0,0-26.5)\end{array}$ \\
\hline \multirow[t]{3}{*}{ Chicken } & Breast meat & 39 & $\begin{array}{c}12 \\
(30.8,17-47.6)\end{array}$ & $\begin{array}{c}5 \\
(41.7,15.2-72.3)\end{array}$ & $\begin{array}{c}7 \\
(58.3,27.7-84.8)\end{array}$ & $\begin{array}{c}0 \\
(0,0-26.5)\end{array}$ \\
\hline & Thigh meat & 39 & $\begin{array}{c}15 \\
(38.5,23.4-55.4)\end{array}$ & $\begin{array}{c}7 \\
(46.7,21.3-73.4)\end{array}$ & $\begin{array}{c}8 \\
(53.3,26.6-78.7)\end{array}$ & $\begin{array}{c}0 \\
(0,0-21.8)\end{array}$ \\
\hline & Intestine & 39 & $\begin{array}{c}16 \\
(41,25.6-57.9)\end{array}$ & $\begin{array}{c}13 \\
(81.3,54.5-96)\end{array}$ & $\begin{array}{c}2 \\
(12.5,1.6-38.3)\end{array}$ & $\begin{array}{c}1 \\
(6.3,0.2-30.2)\end{array}$ \\
\hline Humans & Stool & 246 & $\begin{array}{c}13 \\
(5.3,2.8-8.9)\end{array}$ & $\begin{array}{c}10 \\
(76.9,46.2-95)\end{array}$ & $\begin{array}{c}3 \\
(23.1,5-53.8)\end{array}$ & $\begin{array}{c}0 \\
(0,0-24.7)\end{array}$ \\
\hline & Total & 533 & $\begin{array}{c}114 \\
(21.4,18-25.1)\end{array}$ & $\begin{array}{c}62 \\
(54.4,44.8-63.7)\end{array}$ & $\begin{array}{c}48 \\
(42.1,32.9-51.7)\end{array}$ & $\begin{array}{c}4 \\
(3.5,1-8.7)\end{array}$ \\
\hline
\end{tabular}

* The isolation rate was calculated from the total number of the examined samples

** The isolation rate of each species was calculated from the total number of the isolated Campylobacters 
gastroenteritis in humans in developed and developing countries (Rahimi and Ameri, 2011). Morethan $90 \%$ of the reported Campylobacter infections are caused by $C$. jejuni (NARMS, 2010). For the identification of thermophilic Campylobacter species, catalase test, susceptibility to nalidixic acid and cephalothin and rapid hippurate hydrolysis test were performed. The results showed that most of Campylobacter isolates were resistant to nalidixic acid (Table 1), so that the differentiation between C. jejuni, C. lari and $C$. coli based on the susceptibility to nalidixic acid was difficult. C. jejuni is differentiated by rapid $\mathrm{Na}$ hippurate hydrolysis test. However, this phenotypic distinction is not always accurate because other amino acids or peptides which are transported from the culture media or produced during the incubation can give false-positive results. The judgment on hippurate hydrolysis test is usually based on qualitative criteria which are not reliable and may lead to misinterpretation (Megraud, 1987). Therefore, standardization of hippurate hydrolysis test should be performed by optimizing the turbidity of cell suspension which was set between 0.8 (about MacFarland 6 turbidity) and 1.4 (at least 4 MacFarland) at $450 \mathrm{~nm}$ (Fitzgerald and Nachamkin, 2007). In order to confirm the identification and discrimination of $C$. jejuni, qPCR has been used targeting hip $\mathrm{O}$ (benzoglycine amidohydrolase) which is specific for the hippurate activity and discriminates $C$. jejuni from other Campylobacter species (Englen et al., 2003). The results in Table (1) showed that all 31 biochemically suspected $C$. jejuni isolates were confirmed by qPCR (Figure 1). The specificity of this reaction was characteristic because the primer and probe sets specific for $C$. jejuni did not amplify DNA from $C$. coli positive controls and other positive controls. Accordingly, the conventional culture methods and biochemical reactions were $100 \%$ in accordance with the results of PCR for identification and differentiation of $C$. jejuni. The same results were reported in New Zealand (Klena et al., 2004) and in Egypt (Girgis et al., 2014). The Campylobacter species were isolated from $35.1 \%$ of the examined cloacal swabs. Similarly, the isolation rate of Campylobacter species from chicken cloacal swabs was $(35.9 \%)$ that reported in Great Britain (Jorgensen et al., 2011). Nearly similar isolation rate was $39.2 \%$ in Estonia (Mäesaar et al., 2014) and 38.1\% in Spain (Torralbo et al., 2014). Different studies reported higher prevalence rates of Campylobacter species in chickens (Ellerbroek et al., 2010). Such higher isolation rates could be attributed to the isolation of Campylobacter species from fresh fecal samples on the ground which is suspected to be highly contaminated with Campylobacter species from different sources such as wild birds, rodents and free living pets (Studer et al., 1999). Generally, the variation in Campylobacter species isolation rate between different studies could be attributed to different possible reasons, such as, type of examined samples, location, climate factors, hygienic measures and isolation as well as identification techniques (Jorgensen et al., 2011and Chatur et al., 2014). The prevalence of Campylobacter species in poultry is expected to be high in broilers slaughtered at 35-42 days, while in older chickens, the prevalence decreases reflecting acquired immunity (Kalupahana, et al., 2013). During the current study, the examined samples were collected from chickens at 6 weeks age, explaining the relatively high isolation rate of Campylobacters. Out of 46 Campylobacters isolated from cloacal swabs, $45.7 \%$ were identified as $C$. jejuni (Table 2). Nearly similar percentage of 44.4\% was reported in Italy (Pezzotti et al., 2003). Higher isolation rates of $C$. jejuni were also reported in different studies, in Nigeria (Salihu et al., 2012) and Malaysia (Mansouri-najand et al., 2012). However, lower prevalence rate of $31.4 \%$ was 
obtained in Reunion Island (Henry et al., 2011). The Campylobacter species were isolated from $30.8 \%$ and $38.5 \%$ of the examined breast and thigh meat samples respectively. Comparable the occurrence of Campylobacter in chicken meat was reported in Bosnia (Uzunovic-Kamberovic et al., 2007) and Egypt (Saad, 2014). However, higher isolation rates were obtained by different studies in Iran (Zendehbad et al., 2013) and Poland (Wieczorek et al., 2013). Table (2) showed also that $C$. jejuni was isolated from $41.7 \%$ and $46.5 \%$ of breast and thigh meat samples respectively. The obtained results were lower than Sallam (2007) in Japan. However, Saad (2014) reported that the identification of $C$. jejuni was $6.9 \%$ in thigh meat samples. A large number of Campylobacter species are harbored by the intestinal tract of chicken, especially the ceca and colon. During processing activities, where the intestinal tract may leak or rupture, its contents would be transferred to the skin of carcasses. Chicken skin provides suitable microenvironment for the survival of Campylobacters due to accumulation of water which increases the surface area available for bacterial contamination (Chantarapanont et al., 2003). The isolation rate of Campylobacters from skin samples was $30.8 \%$, of which, $50 \%$ were identified as C. jejuni (Table 2). Different studies have also reported the isolation of Campylobacter species from chicken skin samples, for example, $47.5 \%$ in Egypt (Saad, 2014), 46.6\% in North Germany (Garin et al., 2012) and 60\% in Latvia (Kovalenko et al., 2013). Campylobacter species isolation rate was $41 \%$ from cecal parts (Table 2). Comparable results were also reported by Bester and Essack (2012) and Mäesaar et al. (2014). A previously conducted study reported that Campylobacter species were better detected by direct examination of the intestine than cloacal swabs (Bernadette et al., 2012). Such assumption was based on the fact that cecum is the main colonization site of Campylobacter species in chicken (Silva et al., 2011). Campylobacters remain highly important zoonotic pathogens worldwide. It has been estimated that as few as 500 cells of $C$. jejuni could cause human illness (Yang et al., 2003). For that reason, contamination of food with Campylobacters represents a potential health hazard. The occurrence of Campylobacter species in human stool samples was 5.3\%. This result was nearly similar to $5.8 \%$ (Girgis et al., 2014) and 6.6\% (Zaghloul et al., 2012) in Cairo. Moreover, in Alexandria, 6.4\% (Pazzaglia et al., 1995). C. jejuni were identified in the current study from $76.9 \%$ of the examined human stool samples (Table 2). These results were nearly similar to $70.9 \%$ and $69.3 \%$ reported in Chile (Fernandez et al., 1994) and Romania (Sorokin et al., 2007) respectively.

In conclusion, the relatively high isolation rate of Campylobacters from chicken carcasses during the current study could be attributed to the fact that most of chickens are sold in pluck-shop markets that devoid hygienic measures leading to increase the contamination of slaughtered chicken carcasses with Campylobacters. In addition, the high proportion of chicken contaminated with Campylobacter species in different parts of the carcass pose risk for human Campylobacteriosis. Therefore, control of Campylobacter infection in poultry production is a major public health strategy for prevention of Campylobacteriosis.

\section{REFERENCES}

Bernadette, G. G., Essoh, A. E., Solange, K. E., Natalie, G., Souleymane, B., Sebastien, N. L., Mireille, D. 2012. Prevalence and antimicrobial resistance of thermophilic Campylobacter isolated from chicken in Cote d'Ivoire. Int. J.Microbiol., Vol. 2012, 1-5.

Bester, L.A., Essack, S.Y. 2012. Observational study of the prevalence 
and antibiotic resistance of Campylobacter species from different poultry production systems in KwaZulu-Natal, South Africa. J. Food Prot., 75(1): 154-159.

Chantarapanont, W., Berrang, M., Frank, J.F. 2003. Direct microscopic observation and viability determination of Campylobacter jejuni on chicken skin. J. Food Prot., 6: 2222-2230.

Chatur, Y.A, Brahmbhatt, M.N., Modi, S., Nayak, J.B. 2014. Fluoroquinolone resistance and detection of topoisomerase gene mutation in Campylobacter jejuni isolated from animal and human sources. Int. J. Curr. Microbiol. App. Sci., 3(6) 773783.

EFSA, European Food Safety Authority 2010. Analysis of the baseline survey on the prevalence of Campylobacter in broiler batches and of Campylobacter and Salmonella on broiler carcasses in the EU. Part A: Campylobacter and Salmonella prevalence estimates. EFSA J., 8(03): 1503.

Ellerbroek, L.I., Lienan, J.A., Klein, G. 2010. Campylobacter species in broiler flocks at farm level and the potential for cross-contamination during slaughter. Zoonoses Public Health. 57 (7-8): 81- 88.

Englen, M.D., Ladely, S.R., Fedorka-Cray, P.J. 2003. Isolation of Campylobacter and identification by PCR. Methods Mol. Biol., 216: 109-21.

Fernandez, H., Kahler, K., Salazar, R., Rios, M.A. 1994. Prevalence of thermotolerant species of Campylobacter and their biotypes in children and domestic birds and dogs in southern Chile. Rev. Inst. Med. Trop. Sao Paulo. 36(5): 433-436.

Fitzgerald, C., Nachamkin, I. 2007. Campylobacter and Arcobacter. In Manual of Clinical Microbiology, ed. PR Murray, EJ Baron, JH Jorgensen,
ML Landry, MA Pfaller, pp. 933-46. Washington: ASM Press.

Garin, B., Gouali, M., Wouafo, M., Perchec, A., Thu, P.M., Ravaonindrina, N., Urbès, F., Gay, M., Diawara, A., Leclercq, A., Rocourt, J., Pouillot, R. 2012. Prevalence, quantification and antimicrobial resistance of Campylobacter species on chicken neck-skins at points of slaughter in 5 major cities located on 4 continents. Int. J. Food Microbiol., 157: 102-107.

Girgis, S.A., Rashad, S.S., Othman, H.B., Bassim, H.H., Kassem, N.N., ElSayed, F.M. 2014. Multiplex PCR for Identification and Differentiation of Campylobacter Species and their Antimicrobial Susceptibility Pattern in Egyptian Patients. Int. J. Curr. Microbiol. App. Sci., 3(4): 861-875.

Henry, I., Reichardt, J., Denis, M., Cardinale, E. 2011. Prevalence and risk factors for Campylobacter species in chicken broiler flocks in Reunion Island (Indian Ocean). Prev. Vet. Med., 100 (1): 64-70.

ISO, International Organisation for Standardisation 2006. Microbiology of food and animal feeding stuffs Horizontal method for the detection and enumeration of Campylobacter species Part 1: Detection method. ISO 10272-1:2006: 1-16.

Jorgensen, F., Ellis-Iversen, J., Rushton, S., Bull, S.A., Harris, S.A., Bryan, S.J., Gonzalez, A., Humphrey, T.J. 2011. Influence of Season and Geography on Campylobacter jejuni and C. coli Subtypes in Housed Broiler Flocks Reared in Great Britain. Appl. Environ. Microbiol., 77(11):3741.

Kalupahana, R.S., Kottawatta, K.S., Kanankege, K.S., van Bergen, M.A., Abeynayake, P., Wagenaar, J.A. 2013. Colonization of Campylobacter in broiler chickens and layer hens reared in tropical 
Bacteriological and Molecular Identification of Campylobacter Species in Chickens

climates with low-biosecurity. Appl.

Environ Microbiol., 79: 393-395.

Klena, J.D., Parker, C.T., Knibb, K.J., Ibbitt, C., Devane, P.M.L., Horn, S.T., Miller, W.G., Konkel, M.E. 2004. Differentiation of Campylobacter coli, Campylobacter jejuni, Campylobacter lari, and Campylobacter upsaliensis by a Multiplex PCR Developed from the Nucleotide Sequence of the Lipid A Gene IpxA. J. Clin. Microbiol., 42: 5549- 5557.

Kovalenko, K., Roasto, M., Liepin, E., Mäesaar, M., Hörman, A. 2013. High occurrence of Campylobacter species in Latvian broiler chicken production. Food Control. 29: 188-191.

LaGier, M.J., Joseph, L.A., Passaretti, T.V., Musser, K.A., Cirino, N.M. 2004. A real-time multiplexed PCR assay for rapid detection and differentiation of Campylobacter jejuni and Campylobacter coli. Mol. Cell Probes. 18 (4): 275- 82.

Mäesaar, M., Praakle, K., Meremäe, K., Kramarenko, T., Sõgel, J., Viltrop, A., Muutra, K., Kovalenko, K., Matt, D., Hörman, A., Hänninen, M., Roasto, M. 2014. Prevalence and counts of Campylobacter species in poultry meat at retail level in Estonia. Food Control. 44:72-77.

Mansouri-najand, L., Saleha, A.A., Wai, S.S. 2012. Prevalence of multidrug resistance Campylobacter jejuni and Campylobacter coli in chickens slaughtered in selected markets, Malaysia. Trop. Biomed., 29(2): 231238.

Megraud, F. 1987. Diagnostic bacteriologique des infections à Campylobacter. Rev. Fr. Lab., 156:2-16.

Nachamkin, I. 1999. Campylobacter and Arcobacter. In: Murray PR, Baron EJ, Pfaller, MA, Tenover FC, Yolken RH (eds), Manual of Clinical Microbiology, 7th edition.
Washington D.C: ASM Press, pp: 716-26.

NARMS, National Antimicrobial Resistance Monitoring System for Enteric Bacteria 2010. Human Isolates Final Report. U.S. Department of Health and Human Services, CDC. (Accessed at http://www.cdc. gov/narms/reports.htm)

Oxoid (2006): Oxoid Manual $9^{\text {th }}$ Edition 2006, Compiled by E. Y. Bridson.

Pazzaglia, G., Bourgeois, A.L., Mourad, A.S., Gaafar, T., Diab, A.S., Hebert, A., Churilla, A., Murphy, J.R. 1995. Campylobacter diarrhea in Alexandria, Egypt. J. Egypt Pub. Health Assoc., 70 (3-4): 229-241.

Pezzotti, G., Serafin, A., Luzzi, I., Mioni, R., Milan, M., Perin, R. 2003. Occurrence and resistance to antibiotics of Campylobacter jejuni and Campylobacter coli in animals and meat in northeastern Italy. Int. J. Food Microbiol., 82(3): 281-287.

Rahimi, E., Ameri, M. 2011. Antimicrobial resistance patterns of Campylobacter species isolated from raw chicken, turkey, quail, partridge, and ostrich meat in Iran. Food Control. 22: 11651170 .

Saad, A.E.M. 2014. Zoonotic Importance of Campylobacteriosis at Sharkia Province. A thesis for the degree of master degree, Zoonoses Dep., Fac. Vet. Med., Zagazig Univ.

Salihu, M.D., Junaidu, A.U., Magaji, A.A., Yakubu, Y. 2012. Prevalence and Antimicrobial Resistance of Thermophilic Campylobacter isolates from Commercial Broiler in Sokoto, Nigeria. Research Journal of Veterinary Sciences. 5 (2): 51-58.

Sallam, K.I. 2001. Campylobacter contamination in retailed chicken carcasses from Mansoura, Egypt, and its relation to public health. Alex. J. Vet. Sci., 17 (1). 
Sallam, K.I. 2007. Prevalence of Campylobacter in chicken and chicken by-products retailed in Sapporo area, Hokkaido, Japan. Food Control, 18: 1113-1120.

Silva, J., Leite, D., Fernandes, M., Mena, C., Gibbs, P.A., Teixeira, P. 2011. Campylobacter species as a foodborne pathogen: a review. Front. Microbiol., 2:200.

Sorokin, M., Usein, C.R., Irimia, M., Damian, M. 2007. A laboratory-based survey of Campylobacter infections in Prahova County. Roum. Arch. Microbiol. Immunol., 66 (3-4): 85-89.

Studer, E., Luthy, J., Hubner, P. 1999. Study of the presence of Campylobacter jejuni and C. coli in sand samples from four Swiss chicken farms. Res. Microbiol., 150 (3): 213219.

Torralbo, A., Borge, C., Allepuz, A., García-Bocanegra, I., Sheppard, S.K., Perea, A., Carbonero, A. 2014. Prevalence and risk factors of Campylobacter infection in broiler flocks from southern Spain. Prev. Vet. Med., 114(2):106-113.

Uzunovic-Kamberovic, S., Zorman, T., Heyndrickx, M., Možina, S.S. 2007. Role of poultry meat in sporadic Campylobacter infections in Bosnia and Herzegovina: Laboratory-based Study. Croat. Med. J., 48: 842-851.

Vandepitte, J., Verhaegen, J. 2003. Basic laboratory procedures in clinical bacteriology. Second edition, WHO, Switzerland, pp. 42- 43.

Waino, M., Bang, D. D., Lund, M., Nordentoft, S., Andersen, J. S., Pedersen, K., Madsen, M. 2003. Identification of Campylobacteria isolated from Danish broilers by phenotypic tests and species-specific PCR assays. J. Appl. Microbiol., 95: 649-655.

Wieczorek, K., Kania, I., Osek, J. 2013. Prevalence and antimicrobial resistance of Campylobacter species isolated from poultry carcasses in Poland. J. Food Prot., 76(8): 14511455.

Yang, C., Jiang, Y., Huang, K., Zhu, C., Yin, Y. 2003. Application of realtime PCR for quantitative detection of Campylobacter jejuni in poultry, milk and environmental water, FEMS Immunol. Med. Microbiol., 38 (3): 265-271.

Zaghloul, M.Z., Farouk, N., Galal, Z.A. 2012. Detection of Cambylobacter species in stool samples by new methods in comparison to culture. Life Sci. J., 9(4): 2566-2571.

Zendehbad, B., Arian, A.A., Alipour, A. 2013. Identification and antimicrobial resistance of Campylobacter species isolated from poultry meat in Khorasan province, Iran. Food Control. 32:724-727. 